\title{
Immunisation of children: a sound investment for the Millenium
}

\section{Sanath P Lamabadusuriya ${ }^{1}$}

Sri Lanka Journal of Child Health, 2000; 29: 39-44

(Key words: Immunisation, children)

\section{Historical Background}

In the field of vaccine development Jenner and Pasteur easily stand out as the true pioneers. Although Edward Jenner first attempted systematic immunisation against smallpox in 1796, an entire century actually went by before Louis Pasteur in 1885 administered post exposure rabies vaccine cultured in rabbit spinal cord to a young man severely bitten by a dog. Since then during the last century many other vaccines were developed by researchers $^{1}$ (Table 1).

Table 1 - Milestones in vaccine development

\begin{tabular}{|c|c|c|}
\hline Year & Vaccine & Researcher/Developer \\
\hline 1976 & Smallpox & Jenner \\
\hline 1885 & Rabies & Pasteur \\
\hline 1892 & Cholera & Haffkine \\
\hline 1896 & Typhoid & Wright \\
\hline 1913 & Diphtheria (toxin-toxoid) & Behring \\
\hline 1921 & Tuberculosis (BCG) & Calmette \& Guerin \\
\hline 1923 & Diphtheria (toxoid) & Ramon \& Glenny \\
\hline 1923 & Pertussis & Madsen \\
\hline 1927 & Tetanus & Ramon \& Zoeller \\
\hline 1932 & Yellow fever & Sellard \& Laigret \\
\hline 1937 & Influenza & Salk \\
\hline 1949 & Mumps & Smorodintsev \\
\hline 1954 & Inactivated poilo Salk vaccine (IPV) & Salk \\
\hline 1957 & Live attenuated polio vaccine (OPA) & Sabin \\
\hline 1960 & Measles & Edmonston B (Enders); Schwartz \\
\hline 1962 & Rubella & Weller, Nava \& Parkmann \\
\hline 1966 & Mumps & Weibel, Buynach, Hillemann \& Takahashi \\
\hline 1967 & Rabies & Wiktor \\
\hline 1968 & Meningococcus $\mathrm{C}$ & Gotschlich \\
\hline 1971 & Meningococcus A & Gotschlich \\
\hline 1974 & Varicella & Takahashi \\
\hline 1976 & Hepatitis B & Maupas, Hillemann \\
\hline 1978 & Pneumococcus & \\
\hline 1979 & Hepatities A & Provost \& Hillemann \\
\hline 1980 & Haemophilus Influenza type B & \\
\hline 1984 & Vi Polysaccharide Typhoid Vaccine & \\
\hline
\end{tabular}

Global childhood mortality and the expanded programme of immunization (EPI)

Every year almost 10 million deaths occur in children less than 5 years of age and of them over $99 \%$ are in developing countries and $70 \%$ are caused by measles, pertussis and tetanus in spite of effective vaccines being available for these diseases for many years ${ }^{2}$. It is

${ }^{1}$ Professor of Paediatrics, Faculty of Medicine, University of Colombo important to develop new vaccines but it is even more important to find ways to deliver available vaccines to the poorest children who need them most. Although the development of new vaccines requires research in highincome countries, the provision of vaccines to children in developing, low-income countries depends on financial resources, policy decisions combined with political will and other non-scientific factors. 
Immunisation has substantially reduced child mortality since the WHO began the Expanded Programme on lmmunisation (EPI) in 1974. The coverage with the six EPI vaccines has increased from 5\% in 1974 to over $80 \%$ of children in 25 years.

However, the 2 major causes of deaths in children, pneumonia (2.1 million deaths) and diarrhoeal diseases (2.0 million deaths) cannot be prevented by vaccination in poor countries. In high-income countries new vaccines are being introduced to protect young children from Haemophilus influenzae, Streptococcus pneumoniae and rotavirus and if these vaccines are made available universally there would be a dramatic impact on deaths due to pneumonia and diarrhoea.

In Sri Lanka the EPI programme was formally launched in the late 1970's although the BCG, DPT and oral polio vaccines were available from the 1960s and measles vaccination was introduced island-wide in 1984. As the EPI coverage in Sri Lanka is exceptionally high for a low-income country, deaths due to these diseases have been drastically reduced during the last 3 decades.

\section{Current vaccines}

\section{Tuberculosis}

Almost 2-3 million adults and about 100,000 children die due to tuberculosis each year. The efficacy of different BCG vaccines varies between $0 \%$ and $80 \%$ in different populations ${ }^{3}$. BCG is known to prevent disseminated tuberculosis in children better than pulmonary disease in adults. In Sri Lanka, for this reason, TB meningitis, miliary tuberculosis and bonejoint tuberculosis have become rare in children. Globally as there is a resurgence of TB in HIV infected patients, a more effective vaccine against $\mathrm{TB}$ has become a priority and research is underway. BCG should not be given to anyone with symptomatic HIV infection or AIDS. In Sri Lanka, two problems sometimes encountered with the BCG vaccination are "BCGitis" in the left axilia and lack of uptake of the vaccine. If the BCG scar is absent, the vaccine could be re-administered without a prior tuberculin (Mantoux) test if the child is under 1 year and if tuberculin negative in children over 1 year.

\section{Pertussis}

The locally available vaccine is a whole-cell vaccine which is highly effective and inexpensive; however it is associated with more side effects. In the U.K. in the 1970 s, due to adverse publicity given to side- effects, the vaccine coverage dropped to about $20 \%$ and unimmunised children died of pertussis during outbreaks of it. It continues to cause mortality in unimmunised infants in the U.K. even in 1999 ${ }^{4}$. An acellular vaccine has been developed and its use has been confined to some high-income countries such as Japan because of the cost. The efficacy of acellular vaccines is directly related to the number of antigens it contains, which could vary from one to five.

An absolute contraindication for pertussis vaccination is an adverse reaction such as collapse, shock, fever $>40.5^{\circ} \mathrm{C}$, seizures or episodes of screaming; a progressive neurological illness is also considered to be a contraindication. Children vaccinated against pertussis, may subsequently develop a pertussis-like illness due to adenoviral infection.

\section{Diphtheria}

It is given as a toxoid combined with pertussis and tetanus as DPT. It is well tolerated and side effects such as short-lived local reactions are rare. It is very effective and has almost eradicated the disease from Sri Lanka, except for a few cases of nasal diphtheria.

\section{Tetanus}

It is given as an adsorbed toxoid to pregnant mothers during the first 2 pregnancies which has resulted in the virtual elimination of the scourge of neonatal tetanus from most parts of Sri Lanka. It is also given in the EPI as DPT. Tetanus in children is very rare in Sri Lanka although inadequately immunised adults are still at risk. Booster doses are recommended 5 yearly in children and 10 yearly in adults.

\section{Poliomyelitis}

The live oral polio vaccine (Sabin) has been used in Sri Lanka since the early 1960s. If the cold-chain is maintained it is very effective. In patients with immunedeficiency, the inactivated polio vaccine (Salk) which is injected is preferred, due to the possible risk of vaccine induced paralysis. Poliomyelitis has been eradicated from the USA since 1974 and from the Americas since 1991 and a strategy of national immunisation days (NID) has been successful in reducing wild virus induced poliomyelitis cases to very low levels in many low-income countries. Although the WHO goal of eradicating the disease by year 2000 has not been achieved, some low-income countries such as Sri Lanka are free of poliomyelitis (the last reported case was in 1992). However the WHO would not clear Sri Lanka totally because the disease is widely prevalent in TamilNadu and the potential for spread is obvious because of frequent travel between the regions. Although Sri Lanka conducted N[Ds very successfully in the last few years, the strategy for the future would be sub-national or regional immunisation days in the susceptible areas.

It is of interest to observe that in the USA there are 6-8 vaccine associated paralytic polio cases per year and in 
the absence of natural transmission, injectable polio vaccine (IPV) is being considered for the future.

\section{Measles}

Immunisation has substantially reduced mortality from measles world-wide; however measles kills 1.1 million children less than 5 years of age every year, despite the availability of an effective vaccine 5 . In high-income countries measles vaccine is usually given at $12-15$ months of age because sero conversion rates are higher than in infants; many of these countries use a second dose in older children to increase sero conversion rates. In the U.K. the current recommendation is to give the first dose of MMR (measles, mumps, rubella) vaccine after the first birthday and a second dose just before school entry at 5 years ${ }^{6}$. However, in low-income countries such as Sri Lanka, as many children used to die from measles in infancy (i.e. before 12 months) the vaccine is given at 6-9 months (in Sri Lanka it is given at 9 months).

When Schwartz vaccine is given at 4-8 months, sero conversion rates are lower than after vaccination at 9-11 months and more children get measles. However casefatality rates are lower in the excess cases and the protection against non-measles deaths occurs earlier, so that total mortality is lower with immunisation at 6 months in spite of the lower sero conversion rate $^{7}$. As there is some evidence that the Schwartz measles vaccine reduces mortality from conditions other than measles, the measles vaccination may have to be continued in poor countries even if the disease is eradicated $^{8,9}$. Measles is a potential target for eradication as there is no animal reservoir and the vaccine is very effective. However as measles is very infectious, vaccination rates of over $95 \%$ would be required over many years to achieve that goal.

Measles has made a come-back in Sri Lanka since September 1999. As many vaccinated children have got the disease, a second dose of measles vaccine should preferably be given ideally in the form of MMR vaccine at an appropriate age (e.g. at 3 years). During the recent epidemic as many infants below 9 months have got infected the question of the necessity to advance the age of vaccination has arisen.

\section{Rubella}

Rubella vaccine is given routinely to all children in high-income countries in the form of measles, mumps and rubella (MMR) vaccine at about 15 months of age. It has been in use for nearly 30 years. Sri Lanka experienced an outbreak of congenital rubella syndrome in 1994 and 1995 although the rubella vaccine was available in the private sector for about Rs. 55.00 per dose at that time. It is unfortunate that due to lack of awareness, many unimrnunised mothers who could have afforded the expenditure for the vaccine, delivered babies with congenital rubella syndrome. The Ministry of Health made the rubella vaccine available for females between 11-45 years of age in late 1997. However the strategies used to immunise this section of the population were a failure and over a million doses of the vaccine had to be discarded after the expiry date in late 1999 (personal communication). Therefore new strategies have to be developed in the future to provide effective cover. One such strategy would be to include the MMR vaccine in the national EPI schedule, to be given at 3 years of age.

\section{Japanese encephalitis}

This disease has raised its ugly head in Sri Lanka during the last 15 years. Initially it was prevalent in the North Western and North Central Provinces but later on spread to Eastern, Western and Southern Provinces as well. The current recommendation is to give initially 2 doses of the vaccine 2 weeks apart to children over 1 year of age, a 3rd dose 1 year later and 3 yearly booster doses thereafter.

It is interesting to note that in Thailand where both Japanese encephalitis and dengue fever are prevalent, adults immunised against the former, when infected by the dengue virus, developed a less serious disease with fewer complications ${ }^{10}$. As both are flavi viruses there may be cross-immunity and as there is no effective vaccine against dengue fever as yet, the Japanese encephalitis vaccine may provide an additional beneficial effect for our children.

\section{Other vaccines available in Sri Lanka in the private sector}

\section{Measles, mumps, rubella (MMR)}

The MMR vaccine is widely used and is in the National schedule of most high-income countries and is administered at about 15 months of age. In the U.K., the current recommendation is to give the first dose soon after the first birthday and the second dose before school entry at five years ${ }^{6}$. There are 3 mumps vaccine strains that have been used in the MMR vaccine. These are the Urabe strain, Jeryl-Lynn strain and the Rubini strain. The Urabe strain was withdrawn in 1992 after an association with increased risk of aseptic meningitis ${ }^{11}$. In Singapore the Urabe strain was substituted by the Rubini strain during 1993-95 ${ }^{12}$. However, subsequent disease surveillance revealed that the incidence of mumps had increased during the next 4 years indicating primary vaccination failure. Sharp increases in the incidence of mumps in the 1990s prompted the Swiss Federal Office for Public Health to recommend the use of MMR vaccines containing other mumps virus strains e.g. Jeryl-Lynn, rather than the Rubini strain ${ }^{13}$. As mumps is the commonest cause of viral encephalitis, 
although most cases are mild, and as there is multiorgan involvement such as orchitis, pancreatitis, thyroiditis etc. mumps vaccine should be recommended. An ideal solution for Sri Lanka would be to include the MMR vaccine in the National EPI schedule to be given at about 3 years so that there would be a wider coverage for rubella vaccination and a 2nd dose of measles vaccine would also be routinely given.

\section{Haemophilus}

$H$. influenzae may cause almost as many fatal cases of pneumonia and meningitis as $S$. pneumoniae does. The Haemophilus influenza type B vaccine (Hib conjugate vaccine) has been spectacularly successful in highincome countries in reducing the morbidity and mortality due to meningitis, pneumonia, septicaemia, osteomyelitis and acute epigiotitis ${ }^{14}$. However, it's relatively high cost has precluded its inclusion in the national schedules of low-income countries. In a Gambian study, this vaccine reduced $\mathrm{x}$-'ray proven pneumonia by $20 \%$ and total mortality by $6.1 \%{ }^{15}$.

However, vaccines are required in the future to prevent infections due to nonserotypable (unencapsulated) H.influenzae and types a c d e and $\mathrm{f}$, besides $\mathrm{b}$. The recommended schedule in the U.K is for 3 doses to be given in infancy together with the DPT (all 4 antigens are available in combination) and for a single dose to be given between 12 and 48 months if not vaccinated earlier.

\section{Hepatitis $B$}

Globally hepatitis B causes about 600,000 deaths every year; most are adult deaths from primary hepatic carcinoma caused by hepatitis B infection in childhood $^{16}$. Deaths from hepatitis B could be prevented by immunisation but even USD 1.50 for a course of 3 injections is more expensive than the cost of original 6 EPI vaccines ${ }^{2}$. Since 1991 the WHO has suggested to include it in the routine immunisation schedules of all countries with a high prevalence and over 90 countries have done so ${ }^{16.17}$. However, the vaccine is not given routinely in many very poor countries where the incidence of hepatitis is high because of the expenditure involved. In Sri Lanka it is at present recommended for patients who receive frequent blood transfusions such as thalassaemics and haemophiliacs. In high-income countries where the genetically engineered hepatitis B vaccine has been extensively used, no adverse effects have been reported.

In Sri Lanka, at present the carrier rate of Hepatitis B is not sufficiently high to recommend routine immunisation. It is offered to individuals at risk such as medical officers and paramedics. There is some evidence to suggest that a primary course of immunisation in immunocompetent individuals provides adequate immunity for at least 15 years negating the need for booster doses ${ }^{17}$. Under these circumstances it is worth considering whether this vaccine should be recommended for infants who could afford it, in case the hepatitis B carrier rate in Sri Lanka increases in the future.

\section{Hepatitis $A$}

Although this vaccine is available, it is not recommended for routine use in children. It is recommended for frequent travellers to areas of moderate or high endemicity or where the period of stay exceeds 3 months. It may have a place for patients who receive frequent blood transfusions or blood products such as haemophiliacs and thalassaemics because the hepatitis A virus could be transmitted through blood products.

\section{Pneumococcus}

Pneumococcal infection is estimated to cause 1-2 million deaths a year in children under 5 years in developing countries ${ }^{18}$. It is an important cause of pneumonia, meningitis, otitis media and sinusitis. At present in Sri Lanka it is recommended for patients undergoing splenectomy and it would be of some benefit to patients with nephrotic syndrome. The polysaccharide capsular antigens of $S$. pneumoniae do not stimulate a good antibody response in children less than 2 years, which is the age at which the pneumococcus causes greatest morbidity and mortality. Conjugate pneumococcal vaccines are being developed for use in susceptible age groups but are likely to be unaffordable for use in low-income countries.

\section{Varicella}

An attenuated live varicelia vaccine has been developed and used in Japan for many years and the vaccine was licenced for use in the USA in 1995 where it is recommended for use in children between 12 months to 12 years of age. It has an efficacy of about $90 \%$. In the U.K. it is recommended only for immuno compromised children on a named patient basis ${ }^{6}$. When given to children, the total duration of immunity is uncertain at present and therefore the need for a booster dose later has to be considered. It may be recommended for school children who are scheduled to sit for public examinations and who have not been infected earlier and for sportspersons who have to travel abroad for international competitions.

\section{Meningococcus}

The currently available meningococcal vaccine is effective against serogroups $\mathrm{A}$ and $\mathrm{C}$, which are the prevalent strains in the Middle East and is therefore recommended for travellers to that region. However the 
vaccine is ineffective against Group B organisms which have been responsible for outbreaks in the U.K.

\section{Influenza}

Two types of influenza virus are responsible for most clinical illness; influenza A and influenza B. Outbreaks and epidemics are more common with influenza A as experienced locally in 1998. Influenza A viruses are antigenically labile due to changes in the principal surface antigens. Minor changes (antigenic drift) occur progressively seasonally whilst major changes (antigenic shift) occur due to acquisition of new antigens and can result in epidemics or pandemics. Therefore in the U.K. influenza vaccine is prepared each year taking into account the strains of viruses that are currently prevalent ${ }^{6}$. Although an influenza vaccine is available locally it is not recommended for routine use for the above reasons.

\section{Vaccines for the future}

\section{Malaria}

As the parasite has developed resistance to several antimalarial drugs and the mosquito has become resistant to insecticides, the morbidity and mortality rates of malaria keep on increasing worldwide. Production of an effective vaccine has proved difficult because of the polymorphism displayed by the parasite's proteins. The optimum strategy may be a combination of sporozoite (pre-erythrotic) and merozoite (erythrocytic) vaccines ${ }^{19}$.

\section{Rotavirus}

Rotavirus accounts for 80,000 childhood deaths per year globally, because it is the most important cause of severe diarrhoea in children ${ }^{20}$. In low income countries the most susceptible are young infants in contrast to high-income countries where it occurs later. An oral live attenuated vaccine based on rhesus monkey rotavirus was effective when given at 2, 3 and 4 months of age ${ }^{21}$. It had 50-60\% efficacy against severe diarrhoea in children in Peru, Brazil and USA, The tetravalent rotavirus vaccine was licenced in the USA in the 1998 and in the European Union in 1999 but routine use has been suspended because it appears to cause intussusception $^{22}$.

\section{Rabies}

In Sri Lanka, pre-exposure prophylaxis is not offered to children and only post-exposure prophylaxis is available after animal bites. In a preliminary study in Vietnam, pre-exposure rabies vaccination in infancy using 2 doses of purified vero cell rabies vaccine at 2 and 4 months of age produced protective antibody concentrations against rabies $^{23}$. In countries such as Sri Lanka where rabies is enzootic, this option may have to be considered in the future.

\section{HIV}

HIV infection. killed 500,000 children in 1997 (and 1.8 million adults). $80 \%$ of these deaths were in Africa. Difficulties are being encountered in developing an effective vaccine and currently Phase 3 trials are in progress.

\section{Strategies for the future}

\section{Green revolution}

Production of functional proteins by genetic engineering of bacteria, yeast, cultured mammalian cells, or large animals is currently in progress but expensive. Hepatitis $B$ vaccine using recombinant DNA is a good example. At present a green revolution is underway with researchers and industry turning to the vegetable kingdom for low-cost large-scale solutions. Plants can be engineered to make foreign proteins either transiently by infection with recombinant plant virus vectors or permanently by making transgenic plant strains. There are plans to make edible vaccines by expressing antigens in fruits and vegetables. A research group headed by Charles Arntzen in USA is currently working towards production of edible vaccines - recombinant proteins expressed in a widely available plant material that does not need cooking, the banana ${ }^{24}$. Therefore, in this 3rd millenium a banana a day may keep the doctor away!

\section{Global Alliance for Vaccines and Immunisations (GAVI)}

The mission of GAVI is "to save children's lives and protect people's health through widespread use of vaccines". Since 1990 a declining proportion of the approximately 130 million children born every year becomes fully immunised with the original six EPI vaccines. While in the early 90 s 4 out of 5 children were fully immunised, in 1998 only three out of four children were reported to receive full immunisation ${ }^{25}$. Although newer vaccines have become available, over 30 million children born every year will not be adequately protected against vaccine preventable diseases. Of those, 25 million live in countries with less than USD 1000 GNP per capita annually. Every year there are 3 million unnecessary vaccine preventable premature deaths. It is considered not only as a health issue but an issue of fundamental equity and human rights. A Global Fund for Children's vaccines has been set up to rectify the problems that have been identified. The Bill and Melinda Gates Foundation has provided the first contribution to the fund through a commitment of 750 million USD over five years ${ }^{26}$. As Sri Lanka has an annual per capita GNP of 810 USD (i.e. $<1000$ USD) 
and a population of 18 million, it fits into the category of low-income countries that would benefit from GAVI to strengthen its national EPI programme by adding new vaccines, for the benefit of our future children.

\section{Acknowledgements}

The author wishes to thank Mrs. Hemamali Pathmathilake for typing the manuscript.

\section{References}

1. Ajjan N,Vaccination 3d ed. Lyon, Pasteur Merieux 1988.

2. Shann F, Steinhoff M C. Vaccines for children in rich and poor countries. Lancet 1999, 354 (Supplement 11): $7-11$.

3. Cohn D L. Use of the Bacille-Calmette-Guerin vaccination for the prevention of tuberculosis: renewed interest in an old vaccine. American Journal of Medical Science 1997, 313; 372-76.

4. Ranganathan S, Tasker R, Booy R, Habibi P, Nadel S, Brifto J. Pertussis is increasing in unimunised infants: is a change in policy needed? Archives of Disease in Childhood 1999; 80:297-9.

5. World Health Organisation. The World Health report 1998. Geneva WHO, 1998:62.

6. Salisbury $\mathrm{D} \mathrm{M}$, Begg $\mathrm{N} \mathrm{T}$. Immunisation against infectious diseases London HMSO 1996.

7. Aaby P, Anderson M, Soderma M, Jakobsen M, Gomes J, Fernandes M. Reduced childhood mortality after standard measles vaccination at 4-8 months compared with 9-11 months of age. British Medical Journal 1993; 307:1308-11.

8. Aaby P, Samb B, Simondon F, Coll Seek A M, Knudsen K, Whittle H. Non-specific beneficial effect of measles immunisation: analysis of mortality studies from developing countries. British Medical Journal 1995; 311: 481-5.

9. Shann F. A little bit of measles does you good. British Medical Journal 1999; 319: 4-5.

10. Hoke C H, Nisalak A, Sangawhipa N, et al. Protection against Japanese Encephalitis by inactivated vaccines. New England Journal of Medicine 1988; 319, 608614.

11. Furesz J, Contreras G. Vaccine-related mumps meningitis, Canada. Canadian Diseases Weekly Report 1990, 16:253-4.
12. Goh K T Resurgence of mumps in Singapore caused by the Rubini mumps virus vaccine strain. Lancet 1999; 354:1355-6.

13. Galazka A M, Robertson S E, Kraigher A. Mumps and mumps vaccine. a global review. Bulletin of the World Health Organisation 1999; 77:3-14.

14. Steinhoff M C. Haemophilus Influenzae type b infections are preventable everywhere - Commentary. Lancet 1997; 349:1186-7.

15. Mulholland $\mathrm{K}$, Hilton $\mathrm{S}$, Adegbola $\mathrm{R}$, et al. Randomised trial of Haemophilus influenzae type-b tetanus protein conjugate for prevention of pneumonia and meningitis in Gambian infants. Lancet 1997; 349: 1191-97.

16. UNICEF. The state of the world's children 1999. Oxford: OUP, 1999.

17. Banatvala J. Are booster immunisations needed for llifelong hepatitis B immunity. Lancet 2000; 355: 5615 .

18. World Health Organisation. Vaccine research and development: report of the technical review group meeting. June 9-10, 1997 (WHONRD/GEN/97.01) Geneva. WHO, 1997:32-36.

19. Miller L H, Hoffman S 1. Research toward vaccines against malaria. Natural Medicine 1998, 4 (suppl): $520-4$.

20. Cunliffe N A, Kilgore P E, Bresee J S, Steele A D, Luo N, Hart C A, Glass R 1. Epidemiology of rotavirus diarrhoea in Africa a review to access the need for rotavirus immunisation. Bulletin of the World Health Organisation 1998; 76:525-37.

21. Perez-Schael 1, Guntinas M J, Perez M, et al. Efficacy of the rhesus rotavirus based quadrivalent vaccine in infants and young children in Venezuela. New England Journal of Medicine 1997; 337:1181-7.

22. Anon. RotaShield put on hold after bowel problems. SCRIP Magazine, 2456, 21st July 1999, p. 19.

23. Lang J, Hoa D 0, Gioi N V, Tho L T,Vien N C, Kesmedjian V, Plotkin S A. Randomised feasibility trial of pre-exposure rabies vaccination with DTP-IPV in infants. Lancet 1 997; 349:1663-5.

24. Featherstone C. A green revolution for the clinic? Lancet 1997; 349:1526.

25. WHO Vaccine preventable diseases monitoring systems 1999 (Global summary).

26. GAVI Strategy for sustainable immunisation services (Advanced Draft 8th Feb. 2000) 
